in vivo $35: 3001-3009(2021)$

doi:10.21873/invivo.12595

Review

\title{
Screening Tools for Sarcopenia
}

\author{
HIROKI NISHIKAWA ${ }^{1,2}$, AKIRA ASAI ${ }^{1}$, SHINYA FUKUNISHI ${ }^{1,2}$, TOSHIHISA TAKEUCHI $^{1}$, \\ MASAHIRO GOTO ${ }^{1}$, TAKESHI OGURA ${ }^{1}$, SHIRO NAKAMURA ${ }^{1}$, KAZUKI KAKIMOTO $^{1}$, \\ TAKAKO MIYAZAKI ${ }^{1,2}$, SHUHEI NISHIGUCHI $^{3}$ and KAZUHIDE HIGUCHI ${ }^{1}$
}

\author{
${ }^{1}$ The Second Department of Internal Medicine, Osaka Medical and Pharmaceutical University, Takatsuki, Japan; \\ ${ }^{2}$ The Premier Departmental Research of Medicine, \\ Osaka Medical and Pharmaceutical University, Takatsuki, Japan; \\ ${ }^{3}$ Kano General Hospital, Osaka, Japan
}

\begin{abstract}
The elderly people are characterized by multiple comorbidities, dementia, and are at risk of developing sarcopenia and frailty. Sarcopenia is defined by loss of muscle mass and muscle strength or physical decline. Sarcopenia is a main component of physical frailty. Screening tools for sarcopenia that can be easily determined in daily practice are useful and include the SARC-F screening tool. SARC-F is a questionnaire consisting of five questions: Strength (S), Assistance walking (A), Rising from a chair $(R)$, Climbing stairs $(C)$, and Falls $(F)$ on a scale of 0 to 2 . The recommended cutoff value is $\geq 4$ points. The SARC-F has been shown to correlate well with clinical outcomes in the elderly and various underlying diseases, while it is also true that the SARC-F has its shortcomings such as low sensitivity for sarcopenia. In this review, we mainly outline the SARC-F and mention other screening tools for sarcopenia.
\end{abstract}

The aging of Japan's population continues to accelerate (1). According to the Japanese government report, the aging rate (65 years old or older) was $15.1 \%$ of the total population in 1996 , but it increased significantly to $28.4 \%$ in 2019 (2). In addition, the estimated aging rate as of January 1997 is projected to be $27.4 \%$ in 2025 , but as of 2019 , it has already

This article is freely accessible online.

Correspondence to: Hiroki Nishikawa, MD, Ph.D., the Second Department of Internal Medicine, Osaka Medical and Pharmaceutical University, 2-7, Daigakumachi, Takatsukishi, Osaka, 569-8686, Japan. Tel: +81 726831221, e-mail: nishikawa_6392_0207@yahoo.co.jp

Key Words: Sarcopenia, screening tool, SARC-F, sensitivity, specificity, review. exceeded the projection. The number of people aged 65 or older will continue to increase, and is estimated to reach $33.3 \%$ in 2036 and $38.4 \%$ in 2065 . In order to improve the quality of life (QOL) of the increasing number of elderly people, efforts have been made in the fields of health, medical care, and welfare to extend healthy life expectancy (the period of time during which there are no limitations in daily life), but there are still many issues to be addressed. The elderly people are characterized by multiple comorbidities, dementia, and risk of sarcopenia and frailty (3). In 2019, the Ministry of Health, Labour and Welfare (MHLW) in Japan published the "Guidelines for Health Services Based on the Characteristics of the Elderly, Version 2 ", and announced the strengthening of measures against frailty, especially for elderly people aged 75 or older. The guidelines newly added the implementation of "health checkups for frailty" with the aim of integrating health guidance and care prevention. Specifically, the report has recommended the use of a "questionnaire for the elderly aged 75 or older" focusing on frailty in health checkups and other examinations conducted by municipalities since fiscal 2020. This questionnaire is expected to help prevent and raise awareness of frailty among many elderly people. Early intervention through such efforts will lead to a prolongation in healthy life expectancy. In addition, sarcopenia is a condition that forms the basis of physical frailty (4). Sarcopenia leads to decreased physical activity, and decreased physical activity leads to sarcopenia. Both are involved in frailty, forming the so-called "frailty cycle" (4). On the other hand, the number of people aged 60 or older in the world was estimated to be 600 million in 2000, and is projected to increase to 1.2 billion by 2025 and to 2 billion by 2050. Even if the conservative estimation of the prevalence of sarcopenia, the sarcopenic population is expected to exceed 200 million in the next 40 years (5). 
Therefore, the "enclosure" of patients at risk for sarcopenia is clinically important, and a screening tool that can be easily used in daily practice is useful.

\section{Changes in the Definition of Sarcopenia and the SARC-F as a Screening Tool}

More than 30 years have passed since Rosenberg proposed that aging-related skeletal muscle mass loss be called sarcopenia, from the Greek words "sarco" meaning muscle and "penia" meaning loss (6). Later, Baumgartner used the skeletal muscle mass index (SMI), which is the appendicular skeletal muscle mass of the extremities divided by the square of the height $(\mathrm{m})$, as in the calculation of body mass index (BMI), and defined sarcopenia as a SMI lower than two standard deviations from the mean value (7). However, the significance of defining sarcopenia solely in terms of loss of skeletal muscle mass was reviewed, because it was reported that the loss of muscle strength was more significant than the loss of skeletal muscle mass in the elderly, and that dynapenia, which is an aging-related loss of muscle strength, was more reflective of functional disability and mortality (810). In 2010, the consensus on sarcopenia by the European Working Group on Sarcopenia in Older People (EWGSOP) has developed the concept of sarcopenia to include not only a decrease in skeletal muscle mass but also a decrease in muscle strength and physical performance such as grip strength and walking speed (11). Later, the International Working Group on Sarcopenia (IWGS) in 2011 (12) and the Asian working group for sarcopenia (AWGS) in 2014 (13) provided definitions of sarcopenia. In 2016, the Japanese Society of Hepatology (JSH) published criteria for secondary sarcopenia specific to liver disease (14). This criterion has attracted attention as the world's only criterion for secondary sarcopenia, and is characterized by the omission of age restriction from the viewpoint of disease-related sarcopenia, the omission of gait speed, which is complicated to measure in daily practice, and the reference values of skeletal muscle mass on computed tomography (14). Subsequently, in Japan, the Japanese Association on Sarcopenia and Frailty, the Japan Geriatrics Society, and the National Center for Geriatrics and Gerontology took the lead in creating clinical practice guidelines for sarcopenia in 2017 (15). Recently, the definition of sarcopenia has been updated by EWGSOP second edition (EWGSOP2) (16) and AWGS second edition (AWGS2) (3), and practical guidelines for screening, diagnosis, and management of sarcopenia have been published. EWGSOP2 involves 1) detecting low muscle strength and low skeletal muscle mass and quality to confirm sarcopenia as these are important characteristics of sarcopenia, and identifying sarcopenia severity by physical function decline (gait speed, etc.) and 2) providing clear cutoff values that identify sarcopenia (16). However,
AWGS2 recommends early intervention of nutritional and exercise therapy for people at risk of sarcopenia, using only grip strength or chair stand test to assess sarcopenia as an alternative when it is difficult to measure muscle mass (3). In AWGS2, the threshold for grip strength for males has been raised by $2 \mathrm{~kg}$ to $28 \mathrm{~kg}$. The threshold for grip strength for women in AWGS2 remains at $18 \mathrm{~kg}$. In addition, the standard value for walking speed has been changed from less than $0.8 \mathrm{~m} / \mathrm{s}$ to less than $1 \mathrm{~m} / \mathrm{s}(3)$.

EWGSOP2 also proposes a new algorithm for the detection of sarcopenia. This algorithm uses a pathway for defining sarcopenia using Find-Assess-Confirm-Severity (F-A-C-S), instead of the conventional method of determining sarcopenia by the loss of skeletal muscle mass in addition to decreased walking speed or muscle strength decline. First, muscle strength (grip strength) is assessed if screening is positive or there is clinical suspicion; and if there is muscle weakness, sarcopenia is suspected and targeted for intervention. Next, skeletal muscle mass is assessed, and if a decrease is observed, sarcopenia is confirmed. In addition to a decrease in grip strength and skeletal muscle mass, a decrease in physical performance (gait speed, etc.) is classified as severe sarcopenia (16). AWGS2 also recommends the assessment of sarcopenia using a similar technique (3). The SARC-F is used for the screening of sarcopenia.

The selection of subjects for sarcopenia, either for clinical or research purposes, is extremely important. In the following, the SARC-F is mainly outlined, and other screening tools for sarcopenia are also mentioned.

\section{The SARC-F and International Versatility}

The SARC-F was presented by Morley as a screening tool for sarcopenia at the EU/US Committee on Sarcopenia in the Elderly at the International Conference on Sarcopenia Research (ICSR) held in Orlando in 2012 (17, 18). The SARC-F is a questionnaire consisting of five questions: Strength (S), Assistance walking (A), Rising from a chair (R), Climbing stairs (C), and Falls (F) on a scale of 0 to 2 from "not at all" to "very difficult", and the total score (out of 10) is calculated (17). The recommended cutoff value is $\geq 4$ points (17).

Both EWGSOP2 and AWGS2 recommend the use of the SARC-F for the screening of sarcopenia $(3,16)$. Similarly, the Society for Sarcopenia, Cachexia and Wasting Disorders also recommends the use of the SARC-F (19). On the other hand, during difficult times such as the COVID-19 pandemic, the health care system needs to adapt quickly and efficiently. It is essential that all patients have access to nutritional care as part of their primary health care services, even if social distance measures are taken because of the COVID-19 pandemic. Therefore, for example, in Croatia, a simple nutritional screening tool and practical guidance for nutritional care in 
primary practice were proposed and a tele-medicine process suitable for health care providers has been introduced. This protocol includes the Malnutrition Universal Screening Tool ("MUST"), SARC-F and practical guidance on nutritional interventions for family physicians to identify nutritional risk and loss of muscle mass and function (20). The clinical importance of SARC-F has been acknowledged worldwide.

\section{Sarcopenia Detection Power of the SARC-F, the SARC-calf and the SARC-EBM}

The SARC-F. Several reports have examined the power of the SARC-F to detect sarcopenia as defined by the EWGSOP and AWGS criteria, using receiver operating characteristic (ROC) curves. Woo et al. (21) reported in Asians that ROC analysis for sarcopenia defined by EWGSOP, IWGS, and AWGS had high specificity (0.94-0.99) but low sensitivity (0.038-0.048). The data of Kim et al. (22) in Koreans showed similar results in terms of sensitivity and specificity, and the area under the ROC curves (AUCs) for sarcopenia by AWGS criteria were not so high, 0.618 for men and 0.695 for women. In a comparative study by Yang et al. between the SARC-F and other sarcopenia screening tools, the sensitivity and specificity were similar when using the recommended cutoff value of the SARC-F (4 points), but the AUC was considerably higher, 0.86 for men and 0.90 for women (23). However, Ida et al. reported data from Japanese patients with diabetes mellitus (24). Although the specificity was rather low (0.858 in men and 0.724 in women), the sensitivity was somewhat higher $(0.146$ in men and 0.333 in women) than that of other reports (24). Ida et al. also conducted a meta-analysis and reported a specificity of 0.90 and a sensitivity of 0.21 when data from 12,800 subjects in seven articles were combined (25). Therefore, a positive result on the SARC-F indicates a high rate of sarcopenia, while the sensitivity to the presence of sarcopenia is clearly low. For this reason, it is recommended to check muscle strength when there is clinical suspicion for sarcopenia in EWGSOP2 algorithm, regardless of whether SARC-F is positive or negative (16).

The SARC-calf. AWGS2 recommends screening with either calf circumference (CC), which is known to correlate well with limb skeletal muscle mass and BMI (cut-off value: $<34$ $\mathrm{cm}$ for men and $<33 \mathrm{~cm}$ for women (26), the SARC-F questionnaire, or the SARC-calf, which combines the two, as a trigger for case finding (3). Recent studies have shown a strong correlation between CC and appendicular skeletal muscle mass [correlation coefficient (r): $r=0.78$ (men) and $\mathrm{r}=0.76$ (women)] (27). Using the cut-off value for CC recommended by AWGS2, the AUC for sarcopenia was 0.819 , with a good sensitivity/specificity of $0.804 / 0.718$ (28). The cutoff value for SARC-calf is 11 or higher (max, 20 points). If $\mathrm{CC}$ value is less than the cutoff value, 10 points are added to the SARC-F score to evaluate. CC has been reported to have the highest detection of sarcopenia among arm circumference, arm muscle circumference, CC, arm muscle area, triceps skinfold thickness, waist circumference, and BMI (29). The SARC-calf has the role of compensating for the low sensitivity, which is a problem pointed out when the SARC-F is used alone. In an observational study of 384 patients aged 60 years or older, the sensitivity, specificity, and AUC for sarcopenia as determined by AWGS were $0.295,0.981$, and 0.89 for the SARC-F, compared with $0.607,0.947$, and 0.92 for the SARC-calf (30). In 309 patients with advanced cancer, the sensitivity, specificity, and AUC for sarcopenia as determined by AWGS were 0.321 , 0.907 , and 0.70 for the SARC-F vs. $0.666,0.701$, and 0.75 for the SARC-calf, respectively (31). In a recent metaanalysis, the sensitivity of SARC-calf for sarcopenia was reported to be $0.459-0.572$ and specificity $0.877-0.913$, while the sensitivity of SARC-F for sarcopenia was reported to be $0.289-0.553$ and specificity $0.689-0.889$ (32).

The SARC-EBM. Kurita et al. devised the "SARC-F+EBM" method, which adds "EBM" (Elderly and BMI) to the SARC$\mathrm{F}$ and investigated the degree of improvement in the diagnostic performance of sarcopenia. In addition to the score from the SARC-F questionnaire, this method also takes into account whether the patient is 75 years or older and whether the BMI is less than $21 \mathrm{~kg} / \mathrm{m}^{2}$ (33). Elderly people requiring care with a low BMI show a decline in ADL, swallowing function, and cognitive function, and often consume foods that do not require chewing ability (34). When validated in 959 patients with musculoskeletal diseases, the sensitivity, specificity, and AUC of the SARC-F+EBM diagnostic method were $0.778,0.696$, and 0.824 , respectively, for the diagnosis of sarcopenia. They reported that the sensitivity, specificity, and AUC of the SARC-F questionnaire alone were 0.417 , 0.685 , and 0.557 , respectively, for the diagnosis of sarcopenia, and that the SARC-F+EBM diagnostic method was clearly superior in sensitivity (33).

Validity of the SARC-F. By correlating the SARC-F with other sarcopenia screening tools and health-related indicators, the validity of the SARC-F can be examined. For example, there is a significant correlation between the SARC-F and the Short portable sarcopenia measure (SPSM, see below), which is one of the sarcopenia screening tools (35). In order to confirm the validity of the Japanese version of the SARC-F, Ida et al. examined the correlations with the nursing care prevention checklist, instrumental activity of daily living, intellectual activity, social roles, and selfefficacy for falling, all of which showed significant correlations (24). All the results show the validity of the SARC-F. It is also attractive that the SARC-F requires only 
a questionnaire and does not require measurement of $\mathrm{CC}$ as the SARC-calf does. The low sensitivity of the SARC-F for sarcopenia is problematic because the diagnosis is made by first narrowing down the candidates with a highly sensitive test and then confirming the diagnosis with a highly specific test. However, in light of the above reports, the SARC-F can be a useful tool for sarcopenia in daily practice.

With regard to the low sensitivity of the SARC-F to sarcopenia, this is because the questions are only related to muscle strength and physical performance, and do not include items that reflect skeletal muscle mass, such as weight and BMI. Kim et al. reported that there was no difference in $\mathrm{SMI}$ between the $\mathrm{SARC}-\mathrm{F} \geq 4$ group and the $\mathrm{SARC}-\mathrm{F}<4$ group in women (22). Although the SARC-F is a simple assessment tool, it implies that it is not sufficient for sarcopenia screening. However, the SARC-F is closely related to the future decline in physical functioning, thus the SARC-F has the same significance as the direct assessment of sarcopenia (36). In terms of predictive ability for future decline in physical function, the SARC-F and sarcopenia assessed by EWGSOP, AWGS, and IWGS criteria were almost equal regarding predictive ability (36). On the other hand, the correlation between survival and the SARC-F has also been reported. A meta-analysis of five observational studies reported that the pooled hazard ratio for the SARCF questionnaire positivity and mortality was $1.87(p<0.0001)$ (37). These findings suggest that the SARC-F is very important in daily practice.

\section{The SARC-F, Physical Function and Frailty}

There is a study called the ZEVIOUS study that examines the validity of classifying the level of nursing care in home health care patients based on subjective measures of daily function and sarcopenia. In this study, the relationship between the level of nursing care and self-reported daily living and physical functions in 181 patients receiving home health care was cross-sectionally analyzed (38). In the results, the level of nursing care varied, ranging from support level $1(5.5 \%)$ to care-needs level $5(10.5 \%)$, with care-needs level 2 being the most prevalent $(24.9 \%)$. Lifestyle function was assessed with the World Health Organization Disability Assessment Schedule (WHODAS2.0), which reflects the International Classification of Functioning, Disability and Health (ICF), and physical function was assessed with the SARC-F. The higher the level of nursing care, the lower the level of self-reported life and physical functioning (38). Therefore, the level of nursing care and the SARC-F are significantly positively correlated. The SACR-F is also useful for enclosing frailty patients (39). In a study of 447 elderly patients by Bahat et al., the sensitivity and specificity of SARC-F (cutoff $\geq 1$ point) for diagnosing frailty were 0.914 and 0.449 , respectively. In the case of SARC-F (cutoff $\geq 2$ points), the best balance of sensitivity and specificity was achieved in identifying frailty (sensitivity $=0.741 \mathrm{vs}$. specificity $=0.737, \mathrm{AUC}=0.807$ ) (39). In the case of SARC$\mathrm{F}$ (cutoff $\geq 4$ points), the sensitivity and specificity were 0.462 and 0.926 , respectively (39).

\section{Clinical Significance of the SARC-F According to Diseases}

Several reports have examined the significance of SARC-F in Parkinson's disease. In Parkinson's disease patients, the SARCF has been reported to be a prognostic factor for disability (40) and falls (41). In a study of 60 Parkinson's disease patients, the sensitivity of SARC-F for sarcopenia using EWGSOP2 criteria was as low as 0.231, and the SARC-calf was more useful than the SARC-F (42). In 198 diabetic patients, the SARC-F has been reported to be useful in predicting hospitalization and development of disability (43). In diabetic patients, the SARC$\mathrm{F}$ is also useful in predicting mild cognitive impairment (44), depression (45), overactive bladder (46), and sleep disorders (47). In a study of 127 patients undergoing peritoneal dialysis, the sensitivity and specificity of SARC-F for sarcopenia assessed by AWGS2 were 0.700 and 0.812 , respectively (48). In a study of 717 patients with chronic liver diseases (CLDs), the sensitivity and specificity of SARC-F for sarcopenia assessed by the JSH were 0.65 and 0.68 when the cutoff value of SARC-F was set at 1 , and the predictive ability was better than when the cutoff value of SARC-F was set at 4 (49). In a study of 94 patients with systemic sclerosis, the sensitivity and specificity for sarcopenia assessed by EWGSOP2 were 0.400 and 0.810 for the SARC-F, and 0.533 and 0.848 for the SARCcalf, respectively (50). In a study of 355 patients with chronic heart failure, the sensitivity and specificity of SARC-F for sarcopenia assessed by AWGS were 0.525 and 0.962 , respectively, and in terms of predictive ability on Mini Sarcopenia Risk Assessment (MSRA, described later) for sarcopenia, the MSRA-7 had a sensitivity of 0.924 and a specificity of 0.516 , and the MSRA-5 had a sensitivity of 0.939 and a specificity of 0.573 . The AUCs for the SARC-F, MSRA7, and MSRA-5 were 0.78, 0.74 and 0.77, and MSRA-5 and MSRA-7 were reported to be sensitive screening methods (51). The usefulness of SARC-F in diagnosing sarcopenia in cardiovascular diseases has also been reported (52, 53). In 596 cases of non-valvular atrial fibrillation, the SARC-F has been reported to be useful in predicting mortality (53). A study in 108 gastrointestinal cancers reported a low concordance between the SARC-F and SMI (kappa coefficient=0.2) (54). In a study of 77 cancer patients, a significant correlation between the SARC-F and the Charlson comorbidity index, a prognostic score, was noted (55). The cancer-related cachexia prediction model (cancer cachexia score, CSS) in advanced cancer incorporates the SARC-F, which consists of five items: weight loss, SARC-F, PS, appetite loss, and abnormal biochemistry 
Table I. Screening tools for sarcopenia.

\begin{tabular}{|c|c|c|}
\hline Modality & Assessment method & Notes \\
\hline SARC-F & Five queries ( $0-2$ points in each query) & Cutoff point: $\geq 4$ points (max, 10 points) \\
\hline $\mathrm{CC}$ & Measurement of CC & Cutoff point: $34 \mathrm{~cm}$ (men) and $33 \mathrm{~cm}$ (women) \\
\hline SARC-calf & SARC-F and CC (CC: 10 or 0 points) & Cutoff point: $\geq 11$ points ( $\max , 20$ points) \\
\hline SARC-EBM & SARC-F, age, and BMI & Age: 75 years or older, BMI: $<21 \mathrm{~kg} / \mathrm{m}^{2}$ \\
\hline MSRA-7 & $\begin{array}{l}\text { Age, hospitalization history, activity level, } \\
\text { three dietary items, and weight }\end{array}$ & Range $=0-40$, cutoff value: 30 \\
\hline MSRA-5 & $\begin{array}{l}\text { Age, hospitalization history, activity level, } \\
\text { one dietary item, and weight }\end{array}$ & Range $=0-60$, cutoff value: 45 \\
\hline SPSM & BMI, grip strength, and timed chair stand & International versatility is low. \\
\hline Ishii screening tool & Age, grip strength, and CC & $\begin{array}{l}\text { Range: } 0-145 \text { (men) } / 0-150 \text { (women), } \\
\text { cutoff value: } 105 \text { (men)/120 (women) }\end{array}$ \\
\hline Finger-circle test & $\begin{array}{l}\text { A circle with the index finger and thumb } \\
\text { around the thickest part of the calf }\end{array}$ & $\begin{array}{l}\text { Three groups: "bigger", "just-fits", } \\
\text { and "smaller" }\end{array}$ \\
\hline
\end{tabular}

CC: Calf circumference; BMI: body mass index; MSRA: mini sarcopenia risk assessment; SPSM: short portable sarcopenia measure.

(56). In a study of 187 patients with advanced cancer, the SARC-F was shown to be strongly correlated with cancerrelated fatigue (57). In a study of 256 cancer patients aged 60 years or older, the SARC-F correlated well with impaired instrumental activities of daily living and frailty as well as reduced physical and mental health-related QOL (58). Recently, the relationship between the SARC-F and progression of COVID-19 infection has been reported. In a study of 114 patients with COVID-19 infection, COVID-19 patients with higher risk of sarcopenia as assessed by the SARC-F were demonstrated to be more likely to develop severe disease than those without $(68 \%$ vs. $22 \%, p<0.001)$ (59). Of the 407 patients hospitalized with COVID-19, $22 \%$ had rapid weight loss during hospitalization and $73 \%$ had a SARC-F score of 4 or higher (60). In 152 patients with acute cerebral infarction, the SARC-F was reported to be useful in predicting functional outcome 3 months after stroke onset (61). The SARC-F is also closely correlated with lower limb muscle weakness in acute stroke patients (62). In our study of 1,282 patients with gastrointestinal diseases using the SARC-F, the percentage of patients with SARC-F score of 4 or higher was $17.5 \%$ (57/326) in upper gastrointestinal diseases, $12.0 \%$ $(43 / 357)$ in lower gastrointestinal diseases, $17.3 \%(72 / 416)$ in biliary and pancreatic diseases, and $13.7 \%$ (25/183) in liver diseases, and there was no significant difference among the four groups $(p=0.1235)$ (63). In our multivariate analysis of SARC-F 4 or higher, age, gender, albumin level, lymphocyte count, $\mathrm{C}$ reactive protein, and presence of advanced cancer were independent factors (63).

\section{Other Screening Tools for Sarcopenia}

MSRA. The MSRA is available in two versions: the MSRA7 (range $=0-40)$, which consists of seven items: age, hospitalization history, activity level, three dietary items, and weight, and the MSRA-5 (range=0-60), which includes age, hospitalization history, activity level, weight, and one dietary item (64). In a study of 274 elderly Italian patients, a sensitivity of 0.804 and a specificity of 0.505 for sarcopenia with a cut-off value of 30 in MSRA-7, while a sensitivity of 0.804 and a specificity of 0.505 for sarcopenia with a cut-off value of 45 in MSRA-5, have been reported (64). In a study of 384 elderly people in China, MSRA-7 had a sensitivity of 0.869 and a specificity of 0.396 , whereas the MSRA-5 had a sensitivity of 0.902 and a specificity of 0.706 (23).

SPSM. SPSM is a prediction equation that combines BMI, grip strength, and timed chair stand; it has been used in 998 individuals aged 49-65 years, and robustness has been confirmed by sensitivity analysis (65), but its international versatility is low.

Ishii screening tool. The Ishii screening tool is a method to determine the total score of age, grip strength, and CC [range=0-145 (men)/0-150 (women), cutoff value: 105 (men)/120 (women)] (66). It was developed based on the data of 1,971 elderly Japanese patients. Ishii screening tool has been reported to have high sensitivity and specificity ( 0.849 and 0.882 for men, 0.755 and 0.920 for women) for the diagnosis of sarcopenia in the elderly (66). In a study of 94 patients with systemic sclerosis, the sensitivity, specificity, and AUC of the Ishii screening tool for sarcopenia were $0.867,0.734$, and 0.862 , respectively (50).

Finger-circle test. The finger-circle test is a method of making a circle with the index finger and thumb around the thickest part of the calf and dividing it into three groups: "cannot enclose (bigger)", "just-fits", and "a smaller gap 
(smaller)" (67). Of 1,904 participants (mean age=72.8 years), $53 \%$ were classified as "bigger", $33 \%$ were in "just fits" and $14 \%$ were in "smaller". Relative to "bigger", the results statistically associated with sarcopenia ["just fits"; odds ratio $(\mathrm{OR})=2.4$, "smaller"; OR=6.6]. The results also increased the risk of sarcopenia incidence ("just fits"; $\mathrm{OR}=2.1$, "smaller"; $\mathrm{OR}=3.4$ ). Furthermore, the "smaller" had 2.0- and 3.2-fold elevated risks of requiring long-term care insurance services and mortality, respectively (67). The usefulness of the fingercircle test has also been shown in patients with CLDs (68, 69). In our study of 202 patients with CLDs (56 liver cirrhosis cases), the rates of SMI reduction in "bigger", "justfits", and "smaller" were $9.9 \%(14 / 142), 45.5 \%(15 / 33)$, and $77.8 \%(21 / 27$; overall $p<0.01)$. The percentages of sarcopenia in "bigger", "just-fits", and "smaller" were $3.5 \%$ $(5 / 142), 18.2 \%(6 / 33)$, and $33.3 \%(9 / 27$; overall $p<0.01)$ (68). Screening tools for sarcopenia are listed in Table I.

\section{Final Remarks}

In this review article, screening tools for sarcopenia, with a focus on the SARC-F, were outlined. Although the SARC-F has the limitation of low sensitivity, it is an excellent assessment tool because it has high specificity for sarcopenia, and correlates well with other screening tools, prognosis and the level of nursing care. The SARC-F also plays a role in predicting prognosis in the COVID-19 pandemic. however, assessment tools that combine SARC-F with other factors (SARC-calf, SARC-F+EBM, etc.), Ishii screening test, finger-circle test, etc. are also useful. There are many reports on the usefulness of SARC-F not only in the elderly but also in patients with underlying diseases. Regardless of which screening tool is used, it is important to assess muscle mass and strength and physical function in patients suspicious of having sarcopenia. As the number of sarcopenia patients is expected to increase with the aging population, it is hoped that a simpler and more accurate evaluation tool will become available.

\section{Conflicts of Interest}

The Authors have no conflicts of interest to declare in relation to this study.

\section{Authors' Contributions}

Writing the article: H.N.; Editing and reviewing the article: A.A., S.F., M.G., T.O., T.T., T.M., K.K., S.Na., S.Ni., K.H.; Final approval: all Authors.

\section{References}

1 Nakagawa T, Cho J and Yeung DY: Successful aging in East Asia: Comparison among China, Korea, and Japan. J Gerontol
B Psychol Sci Soc Sci 76(Suppl 1): S17-S26, 2021. PMID: 32324214. DOI: 10.1093/geronb/gbaa042

2 Cabinet Office website. Available at: http://www8. cao.go.jp/kourei/whitepaper/index-w. html [Last accessed on September 30, 2021]

3 Chen LK, Woo J, Assantachai P, Auyeung TW, Chou MY, Iijima K, Jang HC, Kang L, Kim M, Kim S, Kojima T, Kuzuya M, Lee JSW, Lee SY, Lee WJ, Lee Y, Liang CK, Lim JY, Lim WS, Peng LN, Sugimoto K, Tanaka T, Won CW, Yamada M, Zhang T, Akishita $\mathrm{M}$ and Arai H: Asian Working Group for Sarcopenia: 2019 consensus update on sarcopenia diagnosis and treatment. J Am Med Dir Assoc 21(3): 300-307.e2, 2020. PMID: 32033882. DOI: $10.1016 /$ j.jamda.2019.12.012

4 Nishikawa H, Fukunishi S, Asai A, Nishiguchi S and Higuchi K: Sarcopenia and frailty in liver cirrhosis. Life (Basel) 11(5): 399, 2021. PMID: 33925660. DOI: 10.3390/life11050399

5 World Health Organization. Ageing and life course. 2009 [cited 2009 April 30]. Available at: http//www.who.int/ageing/en [Last accessed on September 30, 2021]

6 Epidemiologic and methodologic problems in determining nutritional status of older persons. Proceedings of a conference. Albuquerque, New Mexico, October 19-21, 1988. Am J Clin Nutr 50(5 Suppl): 1121-1235, 1989. PMID: 2816807.

7 Baumgartner RN, Koehler KM, Gallagher D, Romero L, Heymsfield SB, Ross RR, Garry PJ and Lindeman RD: Epidemiology of sarcopenia among the elderly in New Mexico. Am J Epidemiol 147(8): 755-763, 1998. PMID: 9554417. DOI: 10.1093/oxfordjournals.aje.a009520

8 Clark BC and Manini TM: Sarcopenia $=/=$ dynapenia. J Gerontol A Biol Sci Med Sci 63(8): 829-834, 2008. PMID: 18772470. DOI: $10.1093 /$ gerona/63.8.829

9 Newman AB, Kupelian V, Visser M, Simonsick EM, Goodpaster BH, Kritchevsky SB, Tylavsky FA, Rubin SM and Harris TB: Strength, but not muscle mass, is associated with mortality in the health, aging and body composition study cohort. J Gerontol A Biol Sci Med Sci 61(1): 72-77, 2006. PMID: 16456196. DOI: 10.1093/gerona/61.1.72

10 Goodpaster BH, Park SW, Harris TB, Kritchevsky SB, Nevitt M, Schwartz AV, Simonsick EM, Tylavsky FA, Visser $M$ and Newman AB: The loss of skeletal muscle strength, mass, and quality in older adults: the health, aging and body composition study. J Gerontol A Biol Sci Med Sci 61(10): 1059-1064, 2006. PMID: 17077199. DOI: 10.1093/gerona/61.10.1059

11 Cruz-Jentoft AJ, Baeyens JP, Bauer JM, Boirie Y, Cederholm T, Landi F, Martin FC, Michel JP, Rolland Y, Schneider SM, Topinková E, Vandewoude M, Zamboni M and European Working Group on Sarcopenia in Older People: Sarcopenia: European consensus on definition and diagnosis: Report of the European Working Group on Sarcopenia in Older People. Age Ageing 39(4): 412-423, 2010. PMID: 20392703. DOI: 10.1093/ageing/afq034

12 Fielding RA, Vellas B, Evans WJ, Bhasin S, Morley JE, Newman AB, Abellan van Kan G, Andrieu S, Bauer J, Breuille D, Cederholm T, Chandler J, De Meynard C, Donini L, Harris T, Kannt A, Keime Guibert F, Onder G, Papanicolaou D, Rolland Y, Rooks D, Sieber C, Souhami E, Verlaan S and Zamboni M: Sarcopenia: an undiagnosed condition in older adults. Current consensus definition: prevalence, etiology, and consequences. International working group on sarcopenia. J Am Med Dir Assoc 12(4): 249-256, 2011. PMID: 21527165. DOI: 10.1016/j.jamda.2011.01.003 
13 Chen LK, Liu LK, Woo J, Assantachai P, Auyeung TW, Bahyah KS, Chou MY, Chen LY, Hsu PS, Krairit O, Lee JS, Lee WJ, Lee Y, Liang CK, Limpawattana P, Lin CS, Peng LN, Satake S, Suzuki T, Won CW, Wu CH, Wu SN, Zhang T, Zeng P, Akishita $\mathrm{M}$ and Arai H: Sarcopenia in Asia: consensus report of the Asian Working Group for Sarcopenia. J Am Med Dir Assoc 15(2): $95-$ 101, 2014. PMID: 24461239. DOI: 10.1016/j.jamda.2013.11.025

14 Nishikawa H, Shiraki M, Hiramatsu A, Moriya K, Hino K and Nishiguchi S: Japan Society of Hepatology guidelines for sarcopenia in liver disease (1st edition): Recommendation from the working group for creation of sarcopenia assessment criteria. Hepatol Res 46(10): 951-963, 2016. PMID: 27481650. DOI: 10.1111/hepr.12774

15 Sarcopenia Clinical Practice Guidelines. Available at: http://lifescience.co.jp/shop2/index_0165.html [Last accessed on September 30, 2021]

16 Cruz-Jentoft AJ, Bahat $\mathrm{G}$, Bauer $\mathrm{J}$, Boirie $\mathrm{Y}$, Bruyère $\mathrm{O}$, Cederholm T, Cooper C, Landi F, Rolland Y, Sayer AA, Schneider SM, Sieber CC, Topinkova E, Vandewoude M, Visser M, Zamboni M, Writing Group for the European Working Group on Sarcopenia in Older People 2 (EWGSOP2) and the Extended Group for EWGSOP2: Sarcopenia: revised European consensus on definition and diagnosis. Age Ageing 48(1): 16-31, 2019. PMID: 30312372. DOI: 10.1093/ageing/afy169

17 Malmstrom TK and Morley JE: SARC-F: a simple questionnaire to rapidly diagnose sarcopenia. J Am Med Dir Assoc 14(8): 531532, 2013. PMID: 23810110. DOI: 10.1016/j.jamda.2013.05.018

18 Vellas B, Pahor M, Manini T, Rooks D, Guralnik JM, Morley J, Studenski S, Evans W, Asbrand C, Fariello R, Pereira S, Rolland Y, Abellan van Kan G, Cesari M, Chumlea WC and Fielding R: Designing pharmaceutical trials for sarcopenia in frail older adults: EU/US Task Force recommendations. J Nutr Health Aging 17(7): 612-618, 2013. PMID: 23933872. DOI: 10.1007/s12603-013-0362-7

19 Bauer J, Morley JE, Schols AMWJ, Ferrucci L, Cruz-Jentoft AJ, Dent E, Baracos VE, Crawford JA, Doehner W, Heymsfield SB, Jatoi A, Kalantar-Zadeh K, Lainscak M, Landi F, Laviano A, Mancuso M, Muscaritoli M, Prado CM, Strasser F, von Haehling S, Coats AJS and Anker SD: Sarcopenia: A time for action. An SCWD position paper. J Cachexia Sarcopenia Muscle 10(5): 956-961, 2019. PMID: 31523937. DOI: 10.1002/jcsm.12483

20 Krznarić Ž, Bender DV, Laviano A, Cuerda C, Landi F, Monteiro R, Pirlich $\mathrm{M}$ and Barazzoni R: A simple remote nutritional screening tool and practical guidance for nutritional care in primary practice during the COVID-19 pandemic. Clin Nutr 39(7): 1983-1987, 2020. PMID: 32425292. DOI: 10.1016/j.clnu.2020.05.006

21 Woo J, Leung J and Morley JE: Validating the SARC-F: a suitable community screening tool for sarcopenia? J Am Med Dir Assoc 15(9): 630-634, 2014. PMID: 24947762. DOI: 10.1016/j.jamda.2014.04.021

$22 \mathrm{Kim}$ S, Kim M and Won CW: Validation of the Korean version of the SARC-F questionnaire to assess sarcopenia: Korean frailty and aging cohort study. J Am Med Dir Assoc 19(1): 40-45.e1, 2018. PMID: 28843526. DOI: 10.1016/j.jamda.2017.07.006

23 Yang M, Hu X, Xie L, Zhang L, Zhou J, Lin J, Wang Y, Li Y, Han Z, Zhang D, Zuo Y and Li Y: Comparing mini sarcopenia risk assessment with SARC-F for screening sarcopenia in communitydwelling older adults. J Am Med Dir Assoc 20(1): 53-57, 2019. PMID: 29909052. DOI: 10.1016/j.jamda.2018.04.012
24 Ida S, Murata K, Nakadachi D, Ishihara Y, Imataka K, Uchida A, Monguchi K, Kaneko R, Fujiwara R and Takahashi H: Development of a Japanese version of the SARC-F for diabetic patients: an examination of reliability and validity. Aging Clin Exp Res 29(5): 935-942, 2017. PMID: 27832470. DOI: 10.1007/s40520-016-0668-5

25 Ida S, Kaneko R and Murata K: SARC-F for Screening of Sarcopenia Among Older Adults: A meta-analysis of screening test accuracy. J Am Med Dir Assoc 19(8): 685-689, 2018. PMID: 29778639. DOI: 10.1016/j.jamda.2018.04.001

26 Kawakami R, Murakami H, Sanada K, Tanaka N, Sawada SS, Tabata I, Higuchi M and Miyachi M: Calf circumference as a surrogate marker of muscle mass for diagnosing sarcopenia in Japanese men and women. Geriatr Gerontol Int 15(8): 969-976, 2015. PMID: 25243821. DOI: 10.1111/ggi.12377

27 Kawakami R, Miyachi M, Sawada SS, Torii S, Midorikawa T, Tanisawa K, Ito T, Usui C, Ishii K, Suzuki K, Sakamoto S, Higuchi M, Muraoka I and Oka K: Cut-offs for calf circumference as a screening tool for low muscle mass: WASEDA'S Health Study. Geriatr Gerontol Int 20(10): 943-950, 2020. PMID: 32886830. DOI: 10.1111/ggi.14025

28 Chen CY, Tseng WC, Yang YH, Chen CL, Lin LL, Chen FP and Wong AMK: Calf circumference as an optimal choice of four screening tools for sarcopenia among ethnic Chinese older adults in assisted living. Clin Interv Aging 15: 2415-2422, 2020. PMID: 33380792. DOI: 10.2147/CIA.S287207

29 Nishikawa H, Yoh K, Enomoto H, Iwata Y, Sakai Y, Kishino K, Shimono Y, Ikeda N, Takashima T, Aizawa N, Takata R, Hasegawa K, Koriyama T, Yuri Y, Nishimura T, Nishiguchi S and Iijima H: Calf circumference as a useful predictor of sarcopenia in patients with liver diseases. In Vivo 34(5): 25612569, 2020. PMID: 32871785. DOI: 10.21873/invivo.12073

30 Yang M, Hu X, Xie L, Zhang L, Zhou J, Lin J, Wang Y, Li Y, Han Z, Zhang D, Zuo Y, Li Y and Wu L: Screening sarcopenia in community-dwelling older adults: SARC-F vs SARC-F combined with calf circumference (SARC-CalF). J Am Med Dir Assoc 19(3): 277.e1-277.e8, 2018. PMID: 29477774. DOI: 10.1016/j.jamda.2017.12.016

$31 \mathrm{Fu} \mathrm{X}$, Tian Z, Thapa S, Sun H, Wen S, Xiong H and Yu S: Comparing SARC-F with SARC-CalF for screening sarcopenia in advanced cancer patients. Clin Nutr 39(11): 3337-3345, 2020. PMID: 32143888. DOI: 10.1016/j.clnu.2020.02.020

32 Voelker SN, Michalopoulos N, Maier AB and Reijnierse EM: Reliability and concurrent validity of the SARC-F and its modified versions: A systematic review and meta-analysis. J Am Med Dir Assoc 22(9): 1864-1876.e16, 2021. PMID: 34144049. DOI: $10.1016 /$ j.jamda.2021.05.011

33 Kurita N, Wakita T, Kamitani T, Wada O and Mizuno K: SARC$F$ validation and SARC-F+EBM derivation in musculoskeletal disease: The SPSS-OK study. J Nutr Health Aging 23(8): 732738, 2019. PMID: 31560031. DOI: 10.1007/s12603-019-1222-X

34 Furuya H, Kikutani T, Igarashi K, Sagawa K, Yajima Y, Machida R, Tohara T, Takahashi N and Tamura F: Effect of dysphagia rehabilitation in patients receiving enteral nutrition at home nursing care: A retrospective cohort study. J Oral Rehabil 47(8): 977-982, 2020. PMID: 32506544. DOI: 10.1111/ joor. 13030

35 Malmstrom TK, Miller DK, Simonsick EM, Ferrucci L and Morley JE: SARC-F: a symptom score to predict persons with sarcopenia at risk for poor functional outcomes. J Cachexia 
Sarcopenia Muscle 7(1): 28-36, 2016. PMID: 27066316. DOI: $10.1002 / \mathrm{jcsm} .12048$

36 Woo J, Leung J and Morley JE: Defining sarcopenia in terms of incident adverse outcomes. J Am Med Dir Assoc 16(3): 247-252, 2015. PMID: 25548028. DOI: 10.1016/j.jamda.2014.11.013

37 Ida S, Kaneko R, Imataka K, Okubo K, Shirakura Y, Azuma K, Fujiwara R, Takahashi $\mathrm{H}$ and Murata K: Verification of the predictive validity for mortality of the SARC-F questionnaire based on a meta-analysis. Aging Clin Exp Res 33(4): 835-842, 2021. PMID: 32451963. DOI: 10.1007/s40520-020-01585-1

38 Tsugihashi Y, Hirose M, Iida H, Hayashi S, Yasunaka M, Shirahige $\mathrm{Y}$, Kurita $\mathrm{N}$ and ZEVIOUS group: Validating careneeds level against self-reported measures of functioning, disability and sarcopenia among Japanese patients receiving home medical care: The Zaitaku Evaluative Initiatives and Outcome Study. Geriatr Gerontol Int 21(2): 229-237, 2021. PMID: 33393206. DOI: 10.1111/ggi.14124

39 Bahat G, Ozkok S, Kilic C and Karan MA: SARC-F questionnaire detects frailty in older adults. J Nutr Health Aging 25(4): 448-453, 2021. PMID: 33786561. DOI: 10.1007/s12603-020-1543-9

40 Ozer FF, Akın S, Gultekin M and Zararsız GE: Sarcopenia, dynapenia, and body composition in Parkinson's disease: are they good predictors of disability?: a case-control study. Neurol Sci 41(2): 313-320, 2020. PMID: 31583555. DOI: 10.1007/ s10072-019-04073-1

41 Lima DP, de Almeida SB, Bonfadini JC, de Luna JRG, de Alencar MS, Pinheiro-Neto EB, Viana-Júnior AB, Veras SRO, Sobreira-Neto MA, Roriz-Filho JS and Braga-Neto P: Clinical correlates of sarcopenia and falls in Parkinson's disease. PLoS One 15(3): e0227238, 2020. PMID: 32191713. DOI: 10.1371/ journal.pone. 0227238

42 da Luz MCL, Pinho CPS, Bezerra GKA, da Conceição Chaves de Lemos M, da Silva Diniz A and Cabral PC: SARC-F and SARC-CalF in screening for sarcopenia in older adults with Parkinson's disease. Exp Gerontol 144: 111183, 2021. PMID: 33279661. DOI: 10.1016/j.exger.2020.111183

43 Liccini A and Malmstrom TK: Frailty and sarcopenia as predictors of adverse health outcomes in persons with diabetes mellitus. J Am Med Dir Assoc 17(9): 846-851, 2016. PMID: 27569712. DOI: 10.1016/j.jamda.2016.07.007

44 Ida S, Nakai M, Ito S, Ishihara Y, Imataka K, Uchida A, Monguchi K, Kaneko R, Fujiwara R, Takahashi H and Murata K: Association between sarcopenia and mild cognitive impairment using the Japanese version of the SARC-F in elderly patients with diabetes. J Am Med Dir Assoc 18(9): 809.e9-809.e13, 2017. PMID: 28739493. DOI: 10.1016/j.jamda.2017.06.012

45 Ida S, Murata K, Nakai M, Ito S, Malmstrom TK, Ishihara Y, Imataka K, Uchida A, Monguchi K, Kaneko R, Fujiwara R and Takahashi H: Relationship between sarcopenia and depression in older patients with diabetes: An investigation using the Japanese version of SARC-F. Geriatr Gerontol Int 18(9): 13181322, 2018. PMID: 30019805. DOI: 10.1111/ggi.13461

46 Ida S, Kaneko R, Nagata H, Noguchi Y, Araki Y, Nakai M, Ito $\mathrm{S}$, Imataka $\mathrm{K}$ and Murata $\mathrm{K}$ : Association between sarcopenia and overactive bladder in elderly diabetic patients. J Nutr Health Aging 23(6): 532-537, 2019. PMID: 31233074. DOI: 10.1007/s 12603-019-1190-1

47 Ida S, Kaneko R, Nagata H, Noguchi Y, Araki Y, Nakai M, Ito $\mathrm{S}$, Ishihara Y, Imataka K and Murata K: Association between sarcopenia and sleep disorder in older patients with diabetes.
Geriatr Gerontol Int 19(5): 399-403, 2019. PMID: 30773802. DOI: $10.1111 /$ ggi.13627

48 Do JY, Seo JH and Kang SH: Validation of the SARC-F for assessing sarcopenia in patients on peritoneal dialysis. J Ren Nutr, 2021. PMID: 34053820. DOI: 10.1053/j.jrn.2021.03.012

49 Hanai T, Hiraoka A, Shiraki M, Sugimoto R, Taniki N, Hiramatsu A, Nakamoto N, Iwasa M, Chayama K and Shimizu M: Utility of the SARC-F questionnaire for sarcopenia screening in patients with chronic liver disease: a multicenter crosssectional study in Japan. J Clin Med 10(15): 3448, 2021. PMID: 34362231. DOI: $10.3390 / \mathrm{jcm} 10153448$

50 Hax V, do Espírito Santo RC, Dos Santos LP, Farinon M, de Oliveira MS, Três GL, Gasparin AA, de Andrade NPB, Bredemeier M, Xavier RM and Chakr RMDS: Practical screening tools for sarcopenia in patients with systemic sclerosis. PLoS One 16(1): e0245683, 2021. PMID: 33481872. DOI: 10.1371/journal.pone. 0245683

51 Zhao W, Lu M, Wang X and Guo Y: The role of sarcopenia questionnaires in hospitalized patients with chronic heart failure. Aging Clin Exp Res 33(2): 339-344, 2021. PMID: 32346826. DOI: $10.1007 / \mathrm{s} 40520-020-01561-9$

52 Tanaka S, Kamiya K, Hamazaki N, Matsuzawa R, Nozaki K, Maekawa E, Noda C, Yamaoka-Tojo M, Matsunaga A, Masuda $\mathrm{T}$ and Ako J: Utility of SARC-F for assessing physical function in elderly patients with cardiovascular disease. J Am Med Dir Assoc 18(2): 176-181, 2017. PMID: 28043805. DOI: 10.1016/j.jamda.2016.10.019

53 Requena Calleja MA, Arenas Miquélez A, Díez-Manglano J, Gullón A, Pose A, Formiga F, Mostaza JM, Cepeda JM, Suárez $\mathrm{C}$, en nombre de los investigadores del estudio NONAVASC. and Grupo de Riesgo Vascular de la Sociedad Española de Medicina Interna: Sarcopenia, frailty, cognitive impairment and mortality in elderly patients with non-valvular atrial fibrillation. Rev Clin Esp (Barc) 219(8): 424-432, 2019. PMID: 31109685. DOI: $10.1016 /$ j.rce.2019.04.001

54 Siqueira JM, de Oliveira ICL, Soares JDP and Pimentel GD: SARC-F has low correlation and reliability with skeletal muscle mass index in older gastrointestinal cancer patients. Clin Nutr 40(3): 890-894, 2021. PMID: 32907705. DOI: 10.1016/ j.clnu.2020.08.018

55 Borges TC, Gomes TLN and Pimentel GD: Sarcopenia as a predictor of nutritional status and comorbidities in hospitalized patients with cancer: A cross-sectional study. Nutrition 73: 110703, 2020. PMID: 32007693. DOI: 10.1016/j.nut.2019.110703

56 Zhou T, Wang B, Liu H, Yang K, Thapa S, Zhang H, Li L and $\mathrm{Yu}$ S: Development and validation of a clinically applicable score to classify cachexia stages in advanced cancer patients. J Cachexia Sarcopenia Muscle 9(2): 306-314, 2018. PMID: 29372594. DOI: $10.1002 /$ jcsm.12275

57 Wang B, Thapa S, Zhou T, Liu H, Li L, Peng G and Yu S: Cancer-related fatigue and biochemical parameters among cancer patients with different stages of sarcopenia. Support Care Cancer 28(2): 581-588, 2020. PMID: 31102055. DOI: 10.1007/s00520-019-04717-0

58 Williams GR, Al-Obaidi M, Dai C, Bhatia S and Giri S: SARCF for screening of sarcopenia among older adults with cancer. Cancer 127(9): 1469-1475, 2021. PMID: 33369894. DOI: 10.1002/cncr.33395

59 Ma Y, He M, Hou LS, Xu S, Huang ZX, Zhao N, Kang Y, Yue JR and Wu C: The role of SARC-F scale in predicting 
progression risk of COVID-19 in elderly patients: a prospective cohort study in Wuhan. BMC Geriatr 21(1): 355, 2021. PMID: 34112103. DOI: $10.1186 / \mathrm{s} 12877-021-02310-\mathrm{x}$

60 Wierdsma NJ, Kruizenga HM, Konings LA, Krebbers D, Jorissen JR, Joosten MI, van Aken LH, Tan FM, van Bodegraven AA, Soeters MR and Weijs PJ: Poor nutritional status, risk of sarcopenia and nutrition related complaints are prevalent in COVID-19 patients during and after hospital admission. Clin Nutr ESPEN 43: 369-376, 2021. PMID: 34024542. DOI: 10.1016/j.clnesp.2021.03.021

61 Nozoe M, Kanai M, Kubo H, Yamamoto M, Shimada S and Mase K: Prestroke sarcopenia and functional outcomes in elderly patients who have had an acute stroke: A prospective cohort study. Nutrition 66: 44-47, 2019. PMID: 31207438. DOI: 10.1016/j.nut.2019.04.011

62 Nozoe M, Kubo H, Kanai M, Yamamoto M, Shimada S and Mase K: Sarcopenia risk and diabetes mellitus are independent factors for lower limb muscle strength in older patients with acute stroke: A cross-sectional study. Nutrition 84: 111025, 2021. PMID: 33109453. DOI: 10.1016/j.nut.2020.111025

63 Ushiro K, Nishikawa H, Matsui M, Ogura T, Takeuchi T, Goto M, Nakamura S, Kakimoto K, Miyazaki T, Fukunishi S, Asai A, Ohama H, Yokohama K, Yasuoka H and Higuchi K: Comparison of SARC-F score among gastrointestinal diseases. J Clin Med 10(18): 4099, 2021. PMID: 34575208. DOI: 10.3390/jcm10184099

64 Rossi AP, Micciolo R, Rubele S, Fantin F, Caliari C, Zoico E, Mazzali G, Ferrari E, Volpato S and Zamboni M: Assessing the risk of sarcopenia in the elderly: The Mini Sarcopenia Risk Assessment (MSRA) questionnaire. J Nutr Health Aging 21(6): 743-749, 2017. PMID: 28537342. DOI: 10.1007/s12603-0170921-4
65 Miller DK, Malmstrom TK, Andresen EM, Miller JP, Herning MM, Schootman $\mathrm{M}$ and Wolinsky FD: Development and validation of a short portable sarcopenia measure in the African American health project. J Gerontol A Biol Sci Med Sci 64(3): 388-394, 2009. PMID: 19181712. DOI: 10.1093/gerona/gln033

66 Ishii S, Tanaka T, Shibasaki K, Ouchi Y, Kikutani T, Higashiguchi T, Obuchi SP, Ishikawa-Takata K, Hirano H, Kawai H, Tsuji T and Iijima K: Development of a simple screening test for sarcopenia in older adults. Geriatr Gerontol Int 14(Suppl 1): 93-101, 2014. PMID: 24450566. DOI: 10.1111/ggi.12197

67 Tanaka T, Takahashi K, Akishita M, Tsuji T and Iijima K: "Yubiwakka" (finger-ring) test: A practical self-screening method for sarcopenia, and a predictor of disability and mortality among Japanese community-dwelling older adults. Geriatr Gerontol Int 18(2): 224-232, 2018. PMID: 28898523. DOI: 10.1111/ggi.13163

68 Nishikawa H, Yoh K, Enomoto H, Nishimura T, Nishiguchi S and Iijima $\mathrm{H}$ : Clinical impact of the finger-circle test in patients with liver diseases. Hepatol Res 51(5): 603-613, 2021. PMID: 33772972. DOI: 10.1111/hepr.13639

69 Hiraoka A, Izumoto H, Ueki H, Yoshino T, Aibiki T, Okudaira T, Yamago H, Suga Y, Iwasaki R, Tomida H, Mori K, Miyata H, Tsubouchi E, Kishida M, Ninomiya T, Hirooka M, Abe M, Matsuura B, Hiasa Y and Michitaka K: Easy surveillance of muscle volume decline in chronic liver disease patients using finger-circle (yubi-wakka) test. J Cachexia Sarcopenia Muscle 10(2): 347-354, 2019. PMID: 30793533. DOI: 10.1002/jcsm.12392

Received September 12, 2021

Revised October 2, 2021

Accepted October 4, 2021 